\title{
A SURVEY ON NUCLEAR-TO-CYTOPLASMIC RATIO ANALYSIS USING IMAGE SEGMENTATION
}

\author{
S.Karthick ${ }^{1}$, K.Sathiyasekar ${ }^{2}$, S.Lokesh ${ }^{3}$ \\ ${ }^{1}$ Assistant Professor, Department of EEE, The Kavery Engineering College, Salem, India \\ ${ }^{2}$ Professor, Department of EEE, S.A Engineering College, Thiruverkadu, Chennai, India \\ ${ }^{3}$ Post Graduate Student, M.E (Embedded systems), The Kavery Engineering College, Salem, India.
}

\begin{abstract}
In the Bio-medical environment, for the analysis of abnormality detection in the skin including skin cancer in the epidermal layer, the traditional method used is biopsy procedure. The obtained biopsy specimens are processed by various methods viz, various orders of harmonic generations enumerate certain results resulting in the chances of some unexpected infections. Later on to overcome these infections, the followed is optical virtual biopsy procedure with higher harmonics. This yields better results than before. The watershed transform with its gradient works more accurate on the virtual specimens which is observed by microscopically with higher penetration depth.
\end{abstract}

Keywords: - Harmonic generations, Watershed transform, Gradient scale, Morphological processing.

\section{INTRODUCTION}

Image processing involves changing the nature of the images in order to improve the pictorial representation for human interception. In that, image segmentation plays a vital role for further analysis. It involves subdividing an image into constituent parts or isolating certain parts of image. Image segmentation is an valuable tool in many areas which includes industry, health care, radio astronomy, and some other fields too. Segmentation is perfect in a very modest idea. The principal goal of the segmentation process is to partition an image into regions that are homogeneous with respect to one or more characteristics and features. Several methods and approaches are introduced into the area of segmentation, among them a well-known method is watershed algorithm. We can use this algorithm in various fields of life for segmentation of images where we have tried to segment medical images. Medical image segmentation is a very important field for the medical science. In this paper we discussed about the nucleusto-cytoplasm ratio for the analysis abnormality in the human skin. These processes are done by following two major steps viz,

The collection of specimens involves the imaging using microscopic methods with penetration depth in the ranges of micro or even in nano meter called Virtual Biopsy. More the penetration depth, more the accuracy is obtained. Microscopy based on higher order harmonic generated images will help a lot in early diagnosis. Microscopes are essential research tools in biology and medicine. Recently the microscopy images obtained using optical microscopy, which can produce high contrast and high resolution images with the help of laser light source. The ratio between the volume of the Nuclei and the volume of Cytoplasm in the region of interest is said to be $\mathrm{NC}$ Ratio. With the value of the NC ratio obtained from the virtual biopsy, the malignancy can be detected in accordance with the knowledge of the dermatologists.

\section{CELL SEGMENTATION}

The process of cell segmentation and NC ratio analysis constitutes two vital parts viz, nuclei and cytoplasm segmentation. The following sequence depicts the traditional procedure of biopsy.

$\begin{array}{ll}> & \text { Sources of Tissue } \\ > & \text { Freezing } \\ > & \text { Cryomicrotomy } \\ > & \text { Mounting } \\ > & \text { Histochemical reactions } \\ > & \text { Fixation } \\ > & \text { Staining or Counterstaining } \\ > & \text { Cover slipping } \\ > & \text { Microscopy } \\ > & \text { Other analysis }\end{array}$

The above stated steps are followed for the clinical analysis. With these physical biopsy methods, there may be chances for pain to the patients as well as may lead for further infection or even spreading of infected cells.

Later on, to overcome this issue, optical virtual biopsy technique is handled based on Third harmonic generation, which is an electric-dipole process. Based on momentum conservation, mostly the third-harmonic waves are generated in both the forward \& backward direction. Most probably, Backward THG collection (BTHG) may be suitable for 
optically-thick or highly-scattering microscopic samples. With Green's theory, it's proved that the ratio of both THG is a strong material dimension function in thin films and nanoparticles. By this harmonic analysis, it is observed that BTHG signals are able to afford enormous details than Forward THG (FTHG) in nano photonic measurements in calculating the BTHG/FTHG ratios.

\subsection{Nuclei Segmentation}

To specify the nuclei part among the components of the cell. The contextual information of image pixels are obtained by using watershed transform, which is to identify the region minima i.e. nuclei region. Using the gradient map, the boundaries of the nuclei is extracted. For effective horizontal, vertical and positive, negatively sloped diagonal direction, Sobel operator and Roberts gradient operators are employed. This segmentation is a crucial process in forthcoming stages.

\subsubsection{Watershed Transform}

Watershed transform is generally adopted for image segmentation in the area image processing and image analysis. A Watershed is a basin-like landform defined by highpoints and ridgelines that descend into lower elevations and stream valleys. The purpose of watershed transform is not limited in image segmentation, i.e. results generated by watershed transform can be taken as pre-processes for further image analysis. The idea of watershed transform is straightforward by the intuition from geography. Visualize a immersed landscape in a lake, catchment basins with water starting at each local minimum. After reaching the peak water level in the landscape, all catchment basins are alienated by dams, which are called watershed lines. Watershed lines, or briefly speaking watersheds, can divide all the regions (catchment basins) where water will flow.

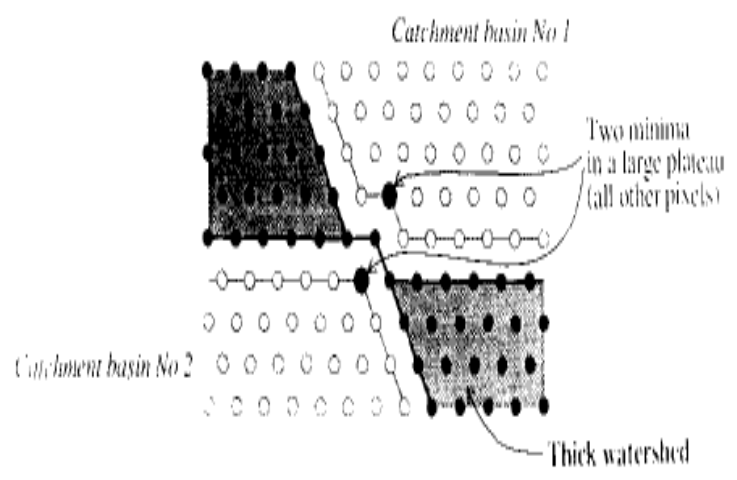

From top to bottom \& right to left

(a) Original image (b) Gradient image

(c) Watershed of Gradient image

(d) Final contour

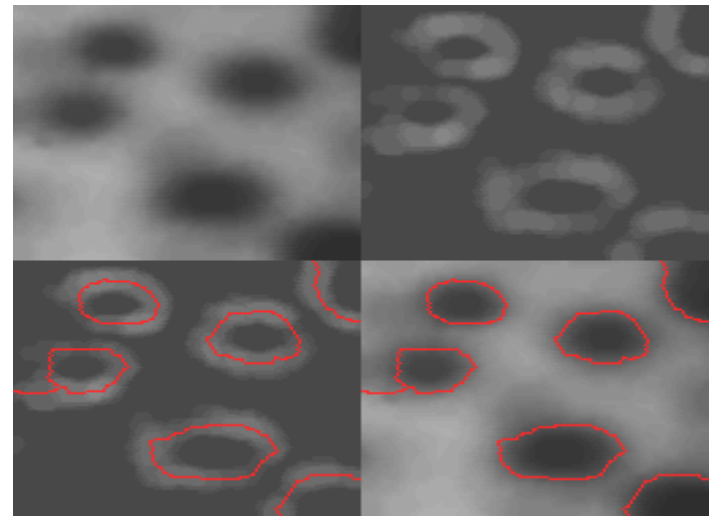

The watersheds can be obtained in two ways viz, boundaries of segmented regions and the segmented regions complement. Watershed algorithms are categorized into two groups. The first is immersion algorithm which simulates the flooding process. This immersion algorithm is one of the most famous watershed segmentation algorithms. It results an efficient way to extract watershed lines by simulating the immersion process on gradient images. The second algorithm aims at direct detection of watershed lines. The watershed algorithm is much apt to segment an image is where the object in an image $s$ overlapped and their borders are very difficult to detect.

When interested objects in an image are delimited by intensity discontinuities, to locate the object, intensity gradient based on edges are used. By considering the multiscale image gradient properties, scale based gradient watershed regions are build. The magnitude of gradient intensity minima image occurs within the centre of homogeneous regions in the original image. With increased blurring, the separating edges of homogeneous regions are removed.

\subsubsection{Marker-Controlled Strategy}

In the process of nuclei Initiation, while applying this watershed segmentation algorithm to an image, over segmentation occurs due to the local irregularities in the image.

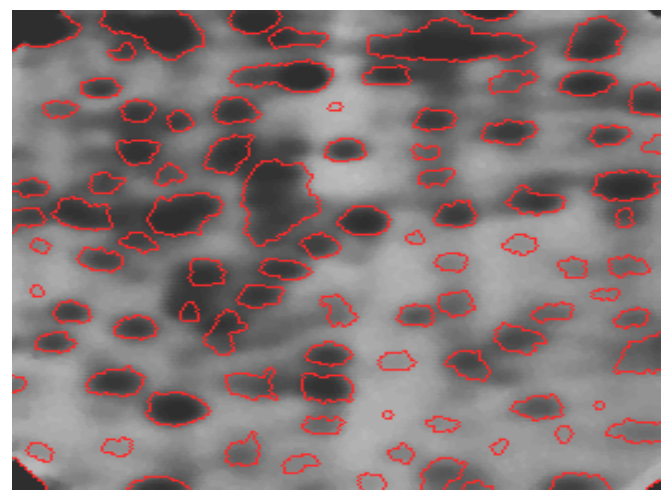

Fig: Marker controlled Watershed Gradient of the image. 
This over segmentation can be avoided by using various markers.

1. Internal Markers: The group of pixels within the region where the potential nuclei is to be segmented. These markers guide the algorithm to fit the size and shape of the nuclei.

2. External Markers: The group of pixels relative to the cellboundary. Over-flooding can prevented by these external markers.

\subsection{Nuclei Initialization}

The objective is to obtain the required nuclei with its appropriate cell boundary by Blob detection. Internal markers need to meet some criteria viz,

- Higher intensity pixels must surround the internal marker region.

- Internal marker region pixels must be connected with homogeneous intensity.

The selected gradient to vary the aims as follows:

a. Noise sensitive.

b. Loss of fine details.

c. Lesser results for watersheds.

d. Direction sequence details.

e. Asymmetrical morphology on maxima and minima image results Black/white symmetry.

f. Shape and size of operator.

g. Computational load.

For marker determination in this nuclei initiation process, of course, the help of medical expert is needed.

\subsubsection{Blob Detection}

In order to extract the interested structures with appropriate scale and spatial information, the original image is spanned into multi-scale representation. Using Hessian matrix, the locality information and scale-space derivatives are obtained. Usually the nuclei are surrounded by enough cytoplasm which is outliers. So those are removed by using local constraints with respect to the blob size.

\subsubsection{Distance Transform}

The external marker approximately gives the boundary of the cell for marker mapping strategy. The segmented nuclei region need fairly away from the external markers. i.e. Euclidean distance which depicts a useful illustration in the cell segmentation area.

\subsection{Morphological Image Processing}

With these marker mapping strategy and their boundaries, a filter is designed to remove the unwanted minima region and to over-come the over-segmentation issue of watershed transform. This morphological processing can be done by following basic operators including Dilation, Erosion,
Opening and Closing etc. The purpose of the Dilation is to expand the shapes and Filling the gaps, holes and gulfs. Meanwhile, Erosion involves in shrinking the shapes and removing the bridges and branches etc. Whereas, Opening and Closing is the compound operation of erosion followed by dilation and vice versa respectively.

In morphological processing, minima imposition is to limit the minima region for the location of marker mapping pixels.

\subsubsection{Nuclei Validation}

The watersheds of the gradient map filter is calculated by transform to make sure the nuclei segmented accurately. Irregularities associated with cancer cells called compactness. A basic property states cell shapes are nearly circular or elliptical with higher compactness.

\subsection{Cytoplasm Segmentation}

Cytoplasms are the major constituents which surrounds the nucleus. The convergence index filter is used for microscopic images with some unpredicted noise. For the initialization of the index filter, there may be no need of algorithmic technical assistance; instead knowing of some basic biomedical image information is more than enough.

- Cell shape and its nuclei are all convex region.

- Cytoplasm and Nuclei are round about concentric.

The above are some assumptions for convergence index filter. The degree of convergence depicts the role of gradient vectors of interested pixel.

\subsubsection{Cytoplasm Initiation}

For initiation, two constraints Rmin and Rmax (minimum \& maximum distance between inner and outer boundary ) have to determined for the shape and position of applicable nuclei. These constraints not only evade the overlapping but also resolve adjacent cell segmentation problem. If the two constraints are defined, with the help of local filter the corresponding regions are detected.

\subsection{Evaluation}

The cell size and $\mathrm{NC}$ ratio are indicators of malignancy in the skin. This evaluation may be given in accordance with the dermatologist for effective result. This cell segmentation based on microscopic imaging technique not only enhances the speed but also results efficiently.

\section{CONCLUSIONS}

With this enhanced processing speed and very less computation time, it is easy to diagnose the skin abnormalities like cancer or any other cytological malignancy. The $\mathrm{NC}$ ratio analysis by this methodology is quite robust than manual approach. 


\section{REFERENCES}

[1]. Automatic Cell Segmentation and Nuclear-to-Cytoplasmic Ratio Analysis For Third Harmonic Generated Microscopy Medical Images GwoGiun (Chris) Lee, Senior Member, IEEE $<$ Huan-Hsiang Lin, Ming-Rung Tsai Sin-Yo-Chou, WenJeng Lee, Yi-Hua Liao, Chi-Kunang Sun, Fellow, IEEE, and Chun-Fu Chen.

[2]. C.-K. Sun, 'Higher Harmonic generation microscopy", Adv. Biochem. Eng./Biotechnol., vol. 95, pp. 17-56, 2005.

[3]. S-Y. Chen, H-Y Wu, and C.K. Sun,;In vivo harmonic generation biopsy of human skin," J. Biomed. Opt., vol 14, no. 6 s, p.060505, 2009

[4]. F.Meyer,'Topographicdistsnce and watershed lines,; Signal process., vol. 38, pp. 113-125, Jul. 1994

[5]. H. Kobatake and S. Hashimoto,' Convergence index filter for vector fields," IEEE Teans Image Process, vol. 8, no,pp. 1029-1038,1999.

[6]. S.-Y. Chen, et al., "In vivo virtual biopsy of human skin by using noninvasivehigherharmonic generation microscopy" IEEE J. Sel. Top Quant. Electron 16, 478-492 (2010)

[7]. H. J. A. M. Heijmans and A. Toet, Morphological Sampling, Center Math. Comput. Sci., Amsterdam, The Netherlands, Internal Rep. AMR8913,Aug. 1989.

\section{BIOGRAPHIES:}

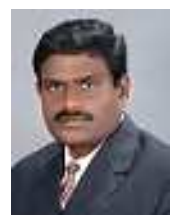

Dr. K. Sathiyasekar, obtained his Ph.D. Degree in High Voltage Engineering, from Anna University, Chennai. He has a total teaching experience of 22 years in various Institutions at B.E and M.E levels. He has published / presented 35 research papers in International Journals / Conferences, and has received the Best Paper Award for his paper titled "Application of BPN Algorithm for Evaluating Insulation Behavior of High Voltage Rotating Machines" in the International Conference on Digital Factory 2008, held at Coimbatore Institute of Technology. He was awarded travel grant by the Department of Science and Technology, Government of India, to present his research paper in the International Conference INDUCTICA - 2010, at Messe Berlin, Germany in 2010.

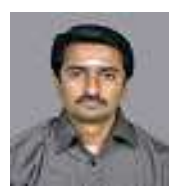

S. Karthick, obtained his Master Degree in Applied Electronics, from Anna University, Chennai. He has 7 years of teaching experience and 6 years in industries. He has presented 14 research papers in International / national Conference. His main area of research is image processing.

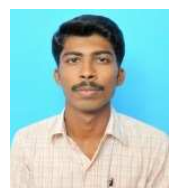

S. Lokesh, A Post Graduate student in The Kavery Engineering College, Salem, India. He obtained his UG degree in Prathyusha Institute of technology \& management, Chennsai, India. 